\title{
LA ACTUALIDAD DEL TRANSGÉNERO EN ESPAÑA Y SU POSIBLE INTERÉS COMO CONTENIDO FORMATIVO
}

\author{
ALLYSON BULLIDO DÍAZ ${ }^{1}$ \\ ANTONIO MATEOS ${ }^{2}$
}

Resumen: El transgénero constituye una de las realidades de la diversidad sexual humana. $\mathrm{Su}$ presencia evidente en la sociedad sigue siendo invisibilizada por los prejuicios y el desconocimiento. El presente artículo relata la situación creada hace unos meses en España y el debate social posterior sobre los niños y niñas con esta condición. El trabajo concluye con opiniones de futuras maestras de Infantil en torno a este asunto y la importancia educativa de trabajar estos contenidos en la formación del profesorado para su traslado al aula.

Palabras clave: Transgénero. Escolares. Formación del profesorado. Educación Infantil.

Resumo: O transgênero constitui uma das realidades da diversidade sexual humana. A sua presença na sociedade continua a ser ocultada por preconceitos e falta de conhecimento. $\mathrm{O}$ presente artigo descreve a situação sobre crianças com esta condição, que surgiu há alguns meses em Espanha, e o debate social subsequente. O estudo apresenta opiniões de futuros/as professores/as sobre este assunto e realça a importância de se trabalhar estes conteúdos na formação de professores/as, no sentido da sua transferência para contextos educativos, nomeadamente o de sala de aula.

Palavras-chave: Transgênero. Escola. Formação de professores/as. Educação infantil.

Abstract: The transgender is one of the realities of human sexual diversity. Its presence in society remains invisible through prejudices and ignorance. This article explores the situation created a few months ago in Spain and the posterior social debate on children with this condition. This essay concludes with the opinions of future early childhood teachers about this particular issue and the educational importance of working with it in teacher training to then transfer it to a class.

Key words: Transgender. Schoolchildren. Teacher training. Early childhood Education.

\section{Introducción}

Durante la infancia, los niños y niñas comienzan a conocerse a sí mismos y a los demás. Para Jennigs (2016) una buena manera de aprender sobre sí mismo es a través de la identificación de su género. Hay niños y niñas que se encuentran cómodos con su sexo e identidad biológica, pero hay otros y otras a los que esta clasificación les produce tristeza,

\footnotetext{
${ }^{1}$ Maestra de Educación Primaria.Toledo, España.

${ }^{2}$ Profesor de la Faculdad de Educación de Toledo. Universidad de Castilla-La Mancha. Área de Didáctica de las Ciencias Experimentales. Grupo GIES.
}

Rev. Diversidade e Educação, v. 5, n. 1, p. 32-41, jan./jun. 2017. 
malestar, ansiedad, etc. porque se sienten insatisfechos con su sexo asignado al nacer, con las características sexuales físicas o con el rol de género asociado con dicho sexo. Existe una gran variedad de maneras de experimentar la masculinidad y la feminidad (Viñuelas \& Guasch, 2000) lo que da lugar a diversas formas de identidad sexual. Una de ellas es la condición transgénero.

El término transgénero se refiere a personas que experimentan una desconexión entre su sexo biológico y su asignación de género ${ }^{3}$. El transgénero fue la autoidentificación para quienes rechazaban las normas de género pero no se identificaban con transexuales o travestis. Hoy en día sirve cómo "término paraguas" para hacer alusión a quienes no aceptan el vínculo entre el sexo biológico y el conjunto de normas de género socialmente aceptadas (Hilton-Morrow \& Battles, 2015). Es suma, transgénero incluye a diversas identidades, entre ellas, transexualidad, travesti o género queer.

\section{Niños y niñas transgénero en España: un debate emergente.}

El asunto del transgénero y de los niños y niñas con esta condición ha irrumpido bruscamente en la sociedad española en los últimos meses. El pasado 18 de febrero de 2017 importantes periódicos nacionales como El País, El Periódico o El Mundo, informaban que un autobús con mensajes contra la diversidad sexual (Fig. 1) recorrería las grandes ciudades del país ${ }^{4}$.

Figura 1

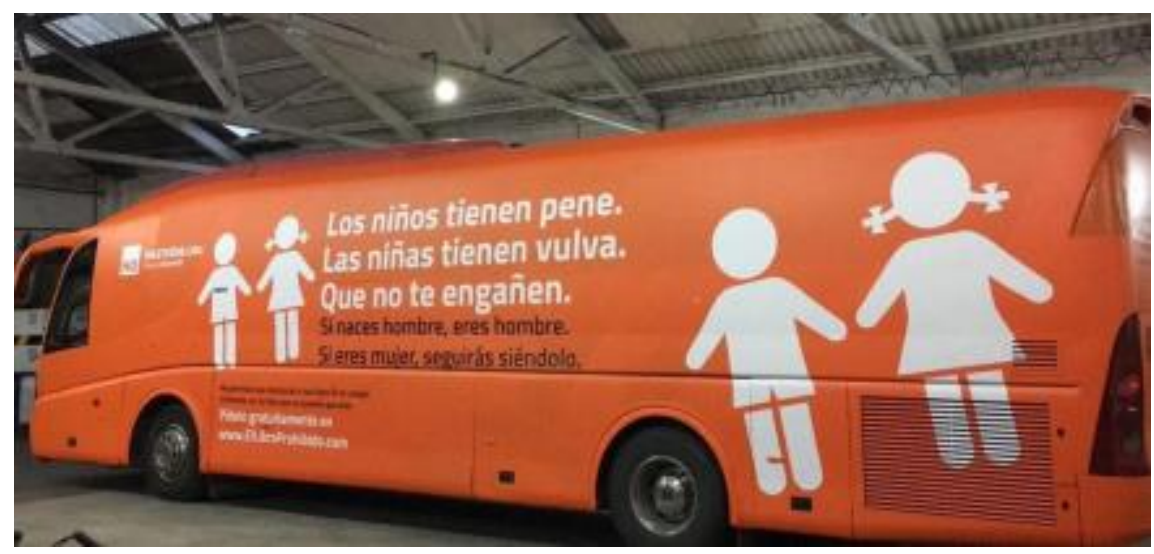

\footnotetext{
${ }^{3}$ Para la American Psychological Association (2011) la identidad de género hace referencia a la experiencia, al sentido interno que una persona tiene de ser hombre, mujer o algo diferente. No confundir con la orientación sexual que hace referencia a la atracción física, romántica y/o emocional de una persona a otra.

${ }^{4} \mathrm{El}$ autobús contra la transexualidad llega este viernes a Barcelona. Extra el Periódico, Barcelona, feb. 2017. Disponible en: <http://www.elperiodico.com/es/noticias/extra/autobus-hazte-oir-contra-transexualidad-llegabarcelona-5865564 >. Acceso en: 20 de Mar. 2017.
}

Rev. Diversidade e Educação, v. 5, n. 1, p. 32-41, jan./jun. 2017. 
HazteOir.org, una asociación española ultra católica, confeccionó un autobús contradiciendo la campaña "Hay niñas con pene y niños con vulva" que la asociación de familias de menores transexuales Chrysallis lanzó a comienzos de este año para concienciar a la sociedad sobre la transexualidad infantil (Fig. 2).

Figura 2

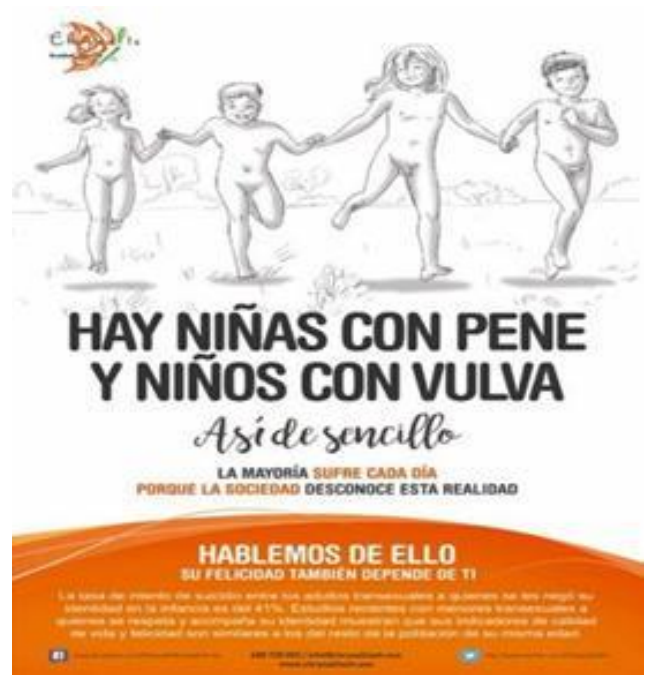

En los laterales del autobús plasmaron el mensaje principal de su campaña: "Los niños tienen pene. Las niñas tienen vulva. Que no te engañen", "Si naces hombre, eres hombre. Si eres mujer, seguirás siéndolo" (Fig. 1). Con estos lemas tránsfobos pretendían hacer publicidad de las afirmaciones que su asociación expone en su folleto: "¿Sabes que quieren enseñarle a tu hijo en el colegio? Las Leyes de adoctrinamiento sexual" (Velázquez, 2017). Según explicó la asociación Hazte Oír a la plataforma "Verne" del periódico El País (Cantó, 2017) diversas copias de este folleto fueron repartidas por numerosos colegios, tanto públicos como privados, de toda España, a finales del año pasado (Cantó, 2016).

El cuadernillo publicado por Hazte Oír, de 44 páginas (Velázquez, 2016), muestra su rechazo hacia el colectivo LGTB (Lesbianas, Gays, Transexuales y Bisexuales) y su desacuerdo con las leyes aprobadas en diversas Comunidades Autónomas españolas que pretenden normalizar la ideología de género y garantizar los derechos de las personas homosexuales, transexuales, etc. y evitar así la discriminación de estos niños y niñas en las aulas. Conviene recordar que las leyes españolas que defienden la ideología de género sancionan las llamadas terapias de aversión o de conversión de orientación sexual e identidad de género. Como señalan Terrasa, Mackintosh y Pinero (2011) la homosexualidad fue eliminada de las categorías de la segunda edición del Manual Rev. Diversidade e Educação, v. 5, n. 1, p. 32-41, jan./jun. 2017. 
Diagnostico y Estadístico de los Trastornos Mentales de la Asociación Norteamericana de Psiquiatría (DSM II). A pesar de ello, el autor de este documento publicado por la asociación ultra-católica, cree que estas leyes quieren ocultar las posibles anomalías y prohibir una terapia concreta. Añade que la proliferación de normas de este tipo premian la conversión de individuos a homosexuales y relegan a los heterosexuales a ciudadanos de segunda categoría. En referencia concreta a la Ley, el autor recalca textualmente:

Evidencia su carácter promocional de la homosexualidad, pues mientras se facilita y premia la conversión de individuos en homosexuales, se niega y castiga la posibilidad en sentido inverso. (VELÁZQUEZ, 2016, p. 27)

Desde la asociación ultra católica también se cuestionan las leyes de identidad de género en el ámbito de la educación. Opina Velázquez (2016) que su introducción en los centros educativos a través de contenidos y materiales didácticos sobre diversidad sexual, diferentes modelos de familia, identidad de género, etc., afecta gravemente a los niños y niñas porque (p. 27):

- Desorientan al imponerle la diversidad sexual.

- Discriminan a la familia natural e imponen modelos familiares.

- Privilegian con dinero público el adoctrinamiento sexual y entregan la educación de los niños y niñas a las organizaciones LGTB.

Entre las reacciones adversas a esta campaña anti transgénero se encuentran la de políticos como la presidenta de la Comunidad de Madrid quien trasladó la posibilidad de que dicha campaña sea contraria a la Ley 2/2016, de 29 de marzo, de Identidad y Expresión de Género e Igualdad Social y no Discriminación de la Comunidad de Madrid (BOCM, 2016). Su artículo 5 expone que ninguna persona podrá ser objeto de exclusión, acoso, penalización o castigo por motivo de su orientación sexual, identidad y/o expresión de género. Igualmente, queda reflejado en dicho artículo normativo la prohibición de cualquier forma de discriminación por razón de identidad o expresión de género o características sexuales (p. 5). Por su parte, el grupo parlamentario socialista solicitó a la

Rev. Diversidade e Educação, v. 5, n. 1, p. 32-41, jan./jun. 2017. 
fiscalía, en el Congreso de los Diputados, que prohibiera circular el autobús por atentar contra los menores transexuales ${ }^{5}$.

Diversas organizaciones ciudadanas también se movilizaron e impulsaron acciones concretas en contra de la iniciativa de Hazte Oír creando en la plataforma de activismo online Change.org una petición apoyada en decenas de miles de firmas para exigir la retirada inmediata del autobús anti transgénero (OLAYA, 2017). Por su parte, grupos de estudiantes de las universidades madrileñas se manifestaron con pancartas a favor de las personas trans y en contra de la transfobia al ver pasar el autobús por su campus (Fig. 3).

Figura 3
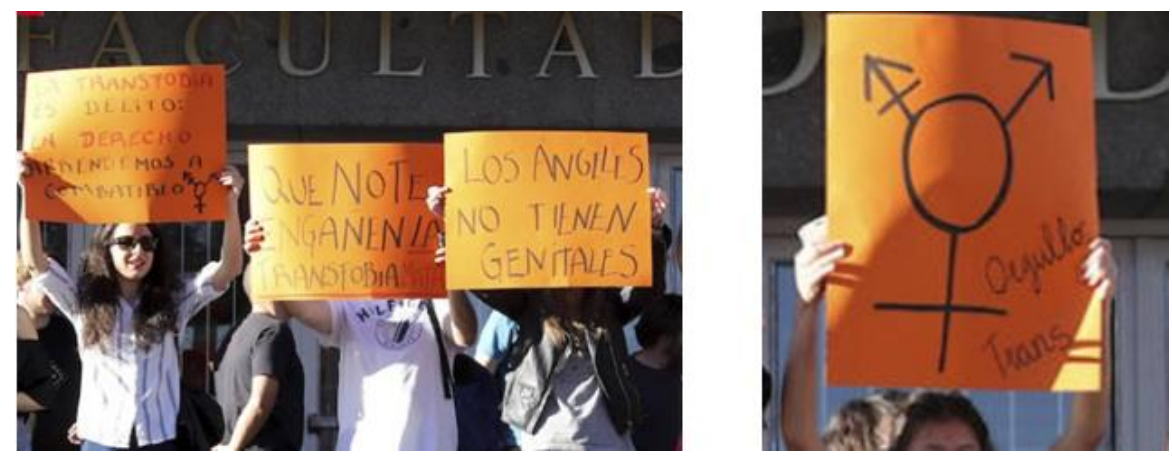

Frente a esta posición a favor de la diversidad sexual, la agencia EFE (EFE, 2017) informó que alrededor de 300 personas se manifestaron en Madrid para apoyar al autobús anti transgénero. El lema de la manifestación era 'Sí a la libertad de expresión. No al adoctrinamiento', y sus objetivos eran defender la libertad de expresión, pedir libertad para poder educar a sus hijos según sus convicciones morales y que no se impusieran los intereses de los grupos minoritarios.

Apesar de los esfuerzos que muchas Comunidades Autónomas españolas están realizando al elaborar leyes para la protección y garantía de derechos de la diversidad sexual y de género así como protocolos de educación y atención sanitaria, existen todavía numerosos actos de odio y fobia hacía las personas trangénero. Este ambiente todavía de resistencia e incomprensión contra la libertad sexual puede contribuir al acoso que sufren los niños y niñas trans y el alto índice de suicidio entre estos menores. Así lo sugieren algunos trabajos con población de origen hispano en California (RYAN, 2010). Estos hechos demuestran la importancia y la necesidad de hacer frente a la violencia sexual desde todos los ámbitos, empezando por el educativo. Para Spalding et al. (2010), la enseñanza y la formación del profesorado tiene en sus manos el martillo de la justicia para

\footnotetext{
${ }^{5}$ http://verne.elpais.com/verne/2017/02/27/articulo/1488212911_849849.html

Rev. Diversidade e Educação, v. 5, n. 1, p. 32-41, jan./jun. 2017.
} 
romper estereotipos y luchar contra la violencia por razón de sexo. Los docentes tienen las herramientas a su disposición para dar este golpe contra las injusticias ya que, a través de sus acciones educativas y sus prácticas diarias, pueden transmitir valores de respeto, tolerancia y aceptación de la diversidad desde las primeras edades y acercarse así a la justicia social. Por todo esto, una buena formación del profesorado constituye una alternativa para luchar por la igualdad.

\section{El transgénero como contenido educativo: primeras impresiones entre un grupo de futuros docentes}

Algunos trabajos revelan que la escuela constituye un lugar clave que genera victimización en los alumnos transgénero y en general en los LGTB (RUSSELL et al., 2010). Ante ello debemos empezar a formar a los estudiantes, futuros docentes, en este contenido. Sin embargo, en ciudades españolas con una fuerte tradición religiosa o católica, este proceso no parece fácil. Interesa saber qué ideas tienen los próximos maestros y maestras sobre los niños y niñas transgénero para ir pulsando cómo formar a estas nuevas generaciones de docentes. Un ejemplo puede encontrarse en la Facultad de Educación de Toledo, ciudad con una gran influencia religiosa y que posee una de las autoridades eclesiásticas más poderosas de España. Para aproximarnos al transgénero y aprovechar la repercusión mediática que produjo la presencia del autobús en la sociedad española, tal y como explicamos anteriormente, abrimos durante esos días (05/03/2017) un asunto específico sobre "Niños y niñas transgénero" en la plataforma académica Moodle, dentro de las unidades curriculares "Biología, Salud y Alimentación Infantil" (BSAI), de 20 del grado de maestro de Educación Infantil, y "Didáctica del Medio Natural, Social y Cultural" (DMNSyC) de $3^{\circ}$ del grado de maestro de Educación Infantil. Los estudiantes debían contestar con libertad y de forma voluntaria dando su opinión en torno a este debate y su problemática. Esta actividad sigue abierta hasta finalizar el curso académico y el cierre de las asignaturas (julio de 2017) y por ello todavía se están obteniendo datos que no permiten adelantar resultados definitivos ya que los estudiantes pueden continuar participando en el foro. Sin embargo, las opiniones que se están detectando revelan, en general, una buena predisposición hacia esta problemática y hacia los escolares que la sufren.

Algunas opiniones que se pueden recoger son:

Rev. Diversidade e Educação, v. 5, n. 1, p. 32-41, jan./jun. 2017. 
NdP (BSAI, 06/03/2017): Respecto a las personas transgénero pienso que debemos tratarlas igual que a los demás, ya que todos somos personas y tenemos los mismos derechos, a pesar de nuestras diferencias. Ni el sexo, ni la raza, ni el origen, ni la religión de una persona debe condicionarnos a la hora de tratarla. En mi opinión, yo pienso que a día de hoy sigue habiendo prejuicios respecto a este colectivo, con lo cual, nosotros como futuros docentes, desde el ámbito educativo, debemos enseñar a los niños desde muy pequeños que todos somos iguales y merecemos el mismo respeto, para en un futuro conseguir que se eliminen los prejuicios hacia estas personas.

MR (BSAI, 06/03/2017I): Este tema ha ocasionado un gran debate. Personalmente, lo más importante es concienciar que todos somos diferentes y hay que respetar las diferencias. Una persona transgénero es igual que nosotros: come, habla, respira...Hay que fomentar el respeto hacia todas las personas sin tener en cuenta el sexo, la mentalidad, ideas, pensamientos, sentimientos, que pertenezca a otro país o que tenga discapacidad.... No importa si un niño o una niña no se siente identificado con su cuerpo. Lo importante es que ese niño o niña se sienta cómodo, arropado y apoyado tanto en la escuela como en la calle. Tiene los mismos derechos que cualquier niño o adulto. Tenemos que dejar a un lado los prejuicios y apoyarles, porque también tienen sentimientos y no son de piedra.

LP (DMNSyC, 10/03/2017): ¡Buenos días! El tema de los niños transgénero o más bien con disforia de género necesita mayor repercusión para que la sociedad comience a dejar sus prejuicios contra ellos. Como futuros profesores debemos tener en cuenta la posibilidad de tener en nuestras aulas niños así y es muy importante que sepamos cómo ayudarlos a ellos y a sus familias para normalizar su situación y que el niño esté plenamente incluido en la escuela y en la sociedad como lo que él o ella se siente y no por lo que digan sus genitales. En mi opinión no debe caber la posibilidad de negarles a estos niños su identidad de género sino que debemos procurar una infancia feliz con un desarrollo personal adecuado para todos los niños de nuestras aulas y eso se logra con la normalización de estas situaciones.

FT (DMNSyC, 14/03/2017): Desde que nacemos sabemos nuestro sexo, pero el género puede que no se corresponda con éste. Por ello como futuros educadores creo que la principal función que tenemos es fomentar el respeto, ya sea hacia este tema o cualquier otro. El objetivo de los creadores de la polémica actual era que en los colegios no se

Rev. Diversidade e Educação, v. 5, n. 1, p. 32-41, jan./jun. 2017. 
impusiese la ideología de género (que se eduque conforme la idea biológica, atendiendo a los órganos genitales), pero desde mi punto de vista si se quiere fomentar el respeto en la sociedad y evitar la discriminación hacia las personas transgénero por donde se debe empezar es en las escuelas. No se debe enseñar que los órganos genitales son los que marcan nuestro género porque cada uno tiene su género coincidiendo o no con los órganos genitales. Por ello, se trabaje como se trabaje, la principal misión será fomentar el respeto hacia la identidad de género.

\section{Conclusiones}

En un mundo como el actual, repleto de avances tecnológicos y conquistas impensables hace años, extraña que la libertad individual para sentirse hombre o mujer (o las dos cosas a la vez) siga en entredicho. Propiciar la libertad de los demás es uno de los principales mandatos que tenemos como ciudadanos de bien. El temor, el miedo y el desconocimiento generan rechazo hacia los demás. Los transgéneros no son seres extraños en una sociedad perfecta. La diversidad sexual que manifiestan es un reflejo más de la naturaleza del ser humano. Parte de la sociedad, por su ignorancia, condena a diario al sufrimiento, a la discriminación y a la exclusión social a las personas cuya sexualidad no concuerda con la biológica. Y si esta realidad se hace agobiante, más aún es en los niños y niñas. Nuestros maestros y maestras deben ser competentes para conseguir individuos libres cuya mente y cuerpo respondan a lo que ellos se sientan en cada momento. Por ello, debemos formar profesorado en estos contenidos esperando que las nuevas generaciones de docentes favorezcan la libertad de las personas transgénero en la escuela.

\section{Referencias:}

AMERICAN PSYCHOLOGICAL ASSOCIATION. Respuestas a sus preguntas sobre las personas trans, la identidad de género y la expresión de género. 2011. Disponible en: <http://www.apa.org/topics/lgbt/transgenero.aspx> Acceso en: 30 abr. 2017.

BOCM. Comunidad Autónoma de Madrid. LEY 2/2016 de Identidad y Expresión de Género e Igualdad Social y no Discriminación de la Comunidad de Madrid. MADRID: BOCM, 2016. p.10.

CANTÓ, Pablo. Identidad sexual: Un autobús de Hazte Oír con un mensaje tránsfobo circula por Madrid. Verne El País, feb. 2017. Disponible en:

Rev. Diversidade e Educação, v. 5, n. 1, p. 32-41, jan./jun. 2017. 
<http://verne.elpais.com/verne/2017/02/27/articulo/1488212911_849849.html >. Acceso en: 20 de Mar. 2017.

CANTÓ, Pablo. Educación: Hazte Oír reparte en colegios un libreto sobre la "amenaza real" de las leyes por diversidad sexual. Verne El País, nov. 2016. Disponible en: <http://verne.elpais.com/verne/2016/11/25/articulo/1480074874_254099.html >. Acceso en: 20 de Mar. 2017.

EFE. Unas 300 personas apoyan el autobús tránsfobo de HazteOir en Madrid. Ideal, Madrid, Mar. 2017. Disponible en: <http://www.ideal.es/sociedad/201703/12/unaspersonas-apoyan-autobus-20170312140047-rc.html >Acceso en: 20 de Mar. 2017.

El polémico autobús de HazteOír que recorre España: "Los niños tienen pene y las niñas vulva. El Mundo, Madrid, Feb.2017. Disponible en:<http://www.elmundo.es/f5/2017/02/28/58b522f4268e3ea2708b4715.html> Acceso en: 20 de Mar. 2017.

HILTON-MORROW, Wendy; BATTLES, Kathleen. Sexual Identities and the Media: An Introduction. New York: Routledge, 2015. 272p.

JENNIGS, Nancy. Soy quien soy: medios, identidad y juventud transexual. Revista Televizion, $\mathrm{n}^{\mathrm{o}}$ Identidad. 2016. Disponible en: <http://www.bronline.de/jugend/izi/spanish/televizion/s_televizion.htm\# > Acceso en: 10 may. 2017.

OLAYA, Felipe. Retirada Inmediata del autobús transfóbico infantil. Change.org, Feb. 2017. Disponible en< https://www.change.org/p/ayuntamiento-de-madrid-retiradainmediata-del-autob\%C3\%BAs-transf\%C3\%B3bico-infantil> Acceso en: 11 de May.2017.

RYAN, Caitlin. Family Acceptance in Adolescence and the Health of LGBT Young Adults. Journal of Child and Adolescent Psychiatric Nursing, v. 23, n. 4, p. 205-213, nov. 2010.

SPALDING, Elizabeth; KLECKA, Cari L.; LIN, Emily; WANG, Jian. Social Justice and Teacher Education: A Hammer, a Bell, and a Song. Journal of Teacher Education, v. 61, n. 3, abr. 2010. Disponible en:

$<$ https://www.researchgate.net/publication/238431033_Social_Justice_and_Teacher_Educ ation_A_Hammer_a_Bell_and_a_Song >. Consultado el 6 de mayo de 2017.

TERRASA, Sergio; MACKINTOSH, Ricardo; PINERO, Agustina. La atención de pacientes lesbianas, gays y bisexuales y transgénero (primera parte). Revista Evidencia actualización en la práctica ambulatoria. v.14, n. 2, p. 57-62, abr/jun. 2011. Disponible en: $<$ http://www.fundacionmf.org.ar/hitalba-paginaarticulo.php?cod_producto=2882\&origen=2 > Acceso en: 27 abr. 2017

Rev. Diversidade e Educação, v. 5, n. 1, p. 32-41, jan./jun. 2017. 
TOOMEY, Russell B.; RYAN, Caitlin; DIAZ, Rafael M.; CARD, Noel A.; RUSSELL, Stephen T. Gender-Nonconforming Lesbian, Gay, Bisexual, and Transgender Youth: School Victimization and Young Adult Psychosocial Adjustment. Developmental Psychology, v. 46, n. 6, p.1580-1589, nov. 2010.

VELÁZQUEZ, Antonio. 2016. ¿Sabes que quieren enseñarle a tu hijo en el colegio?: Las Leyes de adoctrinamiento sexual. 2. ed. Madrid: Hazteoir.org, 2016. 44p.

VIÑUALES, Olga.; GUASCH, Oscar. Sobre el futuro de la identidad. Madrid: Reverso, 2000.

Recebido em: 03/06/2017 Aceito em: 21/09/2017

Rev. Diversidade e Educação, v. 5, n. 1, p. 32-41, jan./jun. 2017. 